\title{
Esophageal Metastasis from Carcinoma Thyroid
}

\section{${ }^{1}$ Arvind Krishnamurthy, ${ }^{2}$ Siddappa KT, ${ }^{3}$ Shirley Sundersingh, ${ }^{4}$ Satish Srinivas, ${ }^{5}$ Krishna Kumar}

${ }^{1}$ Associate Professor, Department of Surgical Oncology, Division of Head and Neck Oncology, Cancer Institute (WIA), Adyar Chennai, Tamil Nadu, India

${ }^{2}$ Postgraduate Student, Department of Surgical Oncology, Division of Head and Neck Oncology, Cancer Institute (WIA), Adyar Chennai, Tamil Nadu, India

${ }^{3}$ Associate Professor, Department of Pathology, Division of Head and Neck Oncology, Cancer Institute (WIA), Adyar, Chennai Tamil Nadu, India

${ }^{4}$ Assistant Professor, Department of Radiotherapy, Division of Head and Neck Oncology, Cancer Institute (WIA), Adyar, Chennai Tamil Nadu, India

${ }^{5}$ Professor, Department of Nuclear Medicine, Division of Head and Neck Oncology, Cancer Institute (WIA), Adyar, Chennai Tamil Nadu, India

Correspondence: Arvind Krishnamurthy, Associate Professor in Surgical Oncology at Cancer Institute, 36, Sardar Patel Road, Adyar, Chennai-20, Tamil Nadu, India, Phone: 9840448174, e-mail: drarvindkrishnamurthy@yahoo.co.in

\section{Abstract}

The incidence of hematogenous spread at the time of presentation of well-differentiated thyroid carcinoma is the range of 4 to $15 \%$. Distant metastases in the most common cause of death from well-differentiated thyroid cancers. About $5 \%$ of papillary carcinoma and $25 \%$ of follicular carcinoma develop distant metastases. Distant metastases occur largely in the lungs and to a lesser extent in the bones, brain and soft tissues. We report and discuss the management of an elderly gentleman with papillary carcinoma thyroid metastatic to the esophagus on follow-up. Only one similar published report is described which was from a follicular carcinoma thyroid. Our case of a papillary carcinoma thyroid metastatic to the esophagus seems to be the first if its kind.

Keywords: Thyroid carcinoma, esophagus, metastasis.

\section{INTRODUCTION}

The incidence of hematogenous spread at the time of presentation of well-differentiated thyroid carcinoma is the range of 4 to $15 \% .{ }^{1}$ A similar spread during the subsequent follow-up period is around $2 \%$ in the low-risk group and $33 \%$ in the high risk group. Distant metastases occur largely in the lungs and to a lesser extent in the bones, brain and soft tissues. We report a case of an elderly gentleman on follow-up for carcinoma thyroid, who presented with dysphagia and on further evaluation, was found to have esophageal metastasis. Only one such published report is available in the literature. ${ }^{2}$

\section{CASE REPORT}

A 63-year-old gentleman was referred to us in July 2000 following a left hemithyroidectomy for a left solitary nodule thyroid. The histopathology slides from the referral center were reviewed by us, which was suggestive of follicular variant of papillary carcinoma thyroid. He then underwent a completion thyroidectomy and central compartment dissection, being in a high-risk group. There was a microscopic focus of papillary carcinoma in the right lobe of the thyroid, the nodes being negative. He was kept on Eltroxin suppression as his local iodine uptake was $1 \%$ and his whole body Iodine 131 scan was negative for metastasis. He was asymptomatic and on regular follow-up till March 2006 when he presented with dysphagia to solids. An endoscopic evaluation revealed a $2 \mathrm{~cm}$ polypoidal lesion in the upper esophagus, $20 \mathrm{~cm}$ from his incisors, a biopsy of which was suggestive of metastasis from papillary carcinoma thyroid. A subsequent evaluation by Iodine scan and a CT Chest scan revealed only the solitary noniodine avid polypoidal lesion in the upper esophagus (Fig. 1). His serum thyroglobulin was $76 \mathrm{ng} / \mathrm{ml}$ and bone scan was normal. The patient refused any further evaluation \{Positron Emission tomography (PET-CT)\} or intervention for the same at that time and insisted on follow-up. His dysphagia progressively worsened over the following three years. The esophageal lesion had grown to $6 \mathrm{~cm}$ and was occupying 


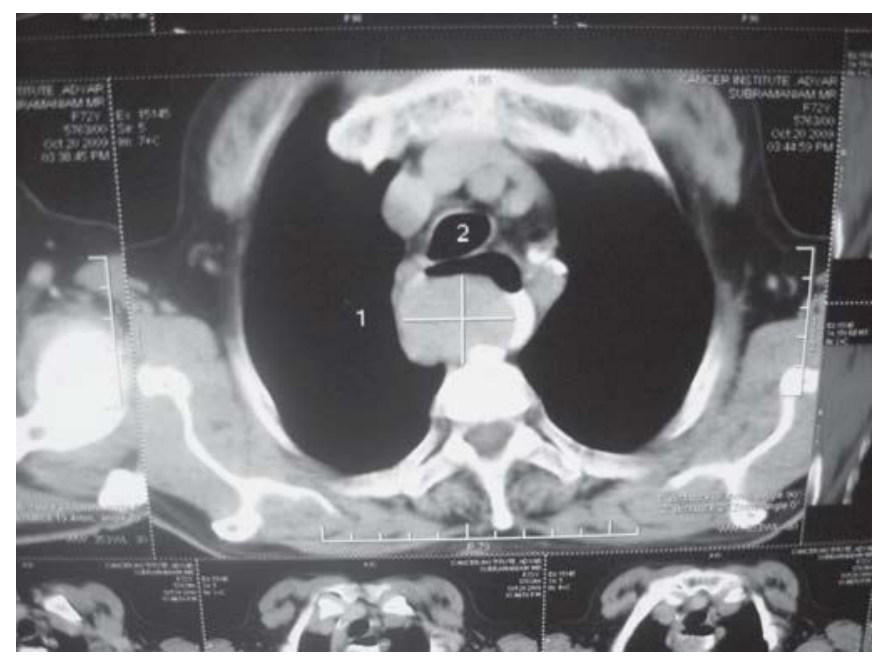

Fig. 1: Polypoidal tumor involving upper esophagus
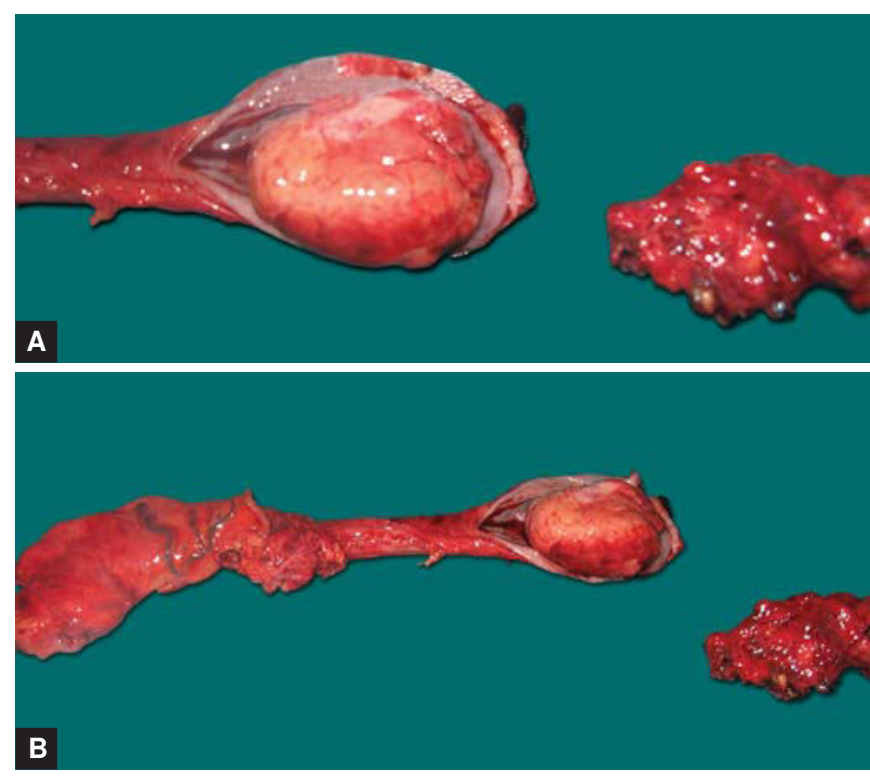

Figs 2A and B: Esophagectomy specimen showing the tumor

more than three fourth of its circumference. An Iodine scan, CT scan and Bone scan were repeated, which confirmed the esophageal lesion as the solitary site of metastasis. The serum thyroglobulin had increased to $153 \mathrm{ng} / \mathrm{ml}$. The patient was planned was surgery after adequate counseling. He underwent an uneventful Trans-Hiatal esophagectomy (Figs 2A and B) in October 2009. The final histopathology was suggestive of a $6 \times 4 \times 2.5 \mathrm{~cm}$ lesion involving the full thickness of the upper esophagus with features suggestive of metastatic papillary carcinoma thyroid, the resected margins and nodes were free. The patient is now asymptomatic, on regular follow-up on Eltroxin suppression with a normal serum thyroglubulin.

\section{DISCUSSION}

Distant metastases in the most common cause of death from well differentiated thyroid cancers. About $5 \%$ of papillary carcinoma and $25 \%$ of follicular carcinoma develop distant metastases.

The most common malignant lesion of the esophagus is primary squamous cell and adenocarcinomas. Esophagus can also be involved by malignant lesions of adjacent structures like larynx, hypopharynx trachea, bronchus and thyroid. The involvement of the esophagus by secondary deposits is a rare occurrence. Such deposits can be asymptomatic $^{3}$ or symptomatic may be due to compression (intramural or submucosal) or intraluminal growth. Metastases from unknown primaries or negative biopsies from submucosal growths pose diagnostic dilemmas. There are a handful of case reports on metastatic involvement of esophagus. The primary in these cases were from the pancreas, ${ }^{4}$ prostate ${ }^{5}$ and from the skin (melanoma). ${ }^{6}$

There is only one case report of a 55 -year-old gentleman with metastatic involvement of esophagus from a follicular carcinoma thyroid. ${ }^{2}$ The presentation was in the form of a polyp which was picked up on a PET- CT scan. Our patient was a much older gentleman of 72 years (when we operated) whose initial primary was papillary carcinoma thyroid. He had symptoms of dyspahgia and on endoscopic evaluation was found to have a pedunculated polyp, which would have been amenable to endoscopic resection initially. However, the polyp had progressed to thrice its initial size during the three year waiting period. An endoscopic resection was not deemed possible in view of the increased size and full thickness involvement of the esophagus. The patient was hence planned for a formal esophagectomy. An esophagectomy by a Trans-Hiatal approach was considered in view of his age and borderline lung function tests. His dysphagia significantly improved after the surgery. He wished to be on follow-up on Eltroxin suppression.

In view of paucity of data, the prognosis of such patients are not clearly known, but is generally expected to be poor.

\section{CONCLUSION}

Carcinoma thyroid with esophageal metastasis is extremely rare. Aggressive management in the form of radical surgery is the best opportunity to provide symptomatic relief and disease control for such patients who in a metastatic setting, especially for lesions that are solitary and not iodine avid. The information on long-term follow-up data as well as on prognosis is very limited. 


\section{REFERENCES}

1. Shaha AR, Shah JP, Loree TR. Differentiated thyroid cancer presenting initially with distant metastasis. Am J Surg 1997; 174:474-76.

2. Lee B, Cook G, John L, Harrington K, Nutting C. Follicular thyroid carcinoma metastasis to the esophagus detected by 18FDG PET/CT. Thyroid 2008 Feb;18(2):267-71.
3. Toreson WE, Arch Path (Lab. Med.), 1944;38,82.

4. Ward P, et al. Pulmonary and esophageal presentations of pancreatic carcinoma. January 1964;37(433).

5. Gross P, Freedman LJ. Arch Path (Lab Med), 1942;33, 36.

6. Eng J, Pradhan GN, Sabanathan S, Mearns AJ. Malignant melanoma metastatic to the esophagus. Ann Thorac Surg Aug 1989;48(2):287-88. 